REVIEW ARTILE

AFRICAN JOURNAL OF CLINICAL AND EXPERIMENT AL MICROBIOLOGY SEPTEMBER 2018 ISBN 1595-689X VOL19 No.4

AJCEM/1841

COPYRIGHT 2018 https://dx.doi.org/10.4314/ajcem.v19i4.9

AFR. J. CLN. EXPER. MICROBIOL. 19 (4):307 -313

\title{
RE-EMERGENCE OF MONKEYPOX IN NIGERIA: A CAUSE FOR CONCERN AND PUBLIC ENLIGHTENMENT
}

\author{
Fowotade, $\mathbf{A}^{1,2}$, Fasuyi TO' ${ }^{1}$ Bakare $\mathbf{R A}^{1,2}$
}

1. Department of Medical Microbiology and Parasitology, University College Hospital, Ibadan, Nigeria; 2. Department of Medical Microbiology and Parasitology, University of Ibadan, Nigeria.

Corresponding Author: Dr Adeola Fowotade. E-mail: temilabike@gmail.com Phone No.: +234-8177557039

\begin{abstract}
Monkey pox infection is a zoonotic infection transmitted by direct or indirect contact with blood, body fluids and lesions of an infected animal. Human to human spread of Monkey pox has been described and infection is usually self-limiting, with an incubation period of 6-16 days. In Nigeria, the last case of monkey pox infection was recorded over 46 years ago. The recent emergence in Nigeria occurred in the year 2017 and was reported to have spread to 24 states with 228 suspected individuals affected. Laboratory diagnosis, as well as management and prevention of monkey pox infection in Nigeria, remain challenging as Nigeria is a resource-poor country with limited infrastructure, technical skill and training which is required in making a diagnosis. The ability of the monkey pox virus to evolve, its potential bioterrorism potential, as well as its recent emergence in Nigeria further justifies the need for improving the understanding of the presentation and prevention of monkey pox infection.
\end{abstract}

Keywords: monkey pox, emerging virus, Nigeria, rash, zoonosis, orthopox

\section{RÉ-ÉMERGENCE DU VIRUS AU NIGERIA: UNE SOURCE D'INQUIÉTUDE ET L'INFORMATION DU PUBLIC}

Fowotade, $\mathbf{A}^{1,2}$, Fasuyi TO', Bakare RA ${ }^{1,2}$

1. Département de microbiologie médicale et de la parasitologie, de l'University College Hospital, Ibadan, Nigeria ; 2. Département de microbiologie médicale et de parasitologie, Université d'Ibadan, Nigeria.

Auteur correspondant : Dr Adeola Fowotade. E-mail : temilabike@gmail.com nº de téléphone : 234-8177557039

Résumé

Infection au virus de la variole du singe est une infection zoonotique transmise par contact direct ou indirect avec du sang, des liquides organiques et des lésions d'un animal infecté. Homme à homme propagation de la variole du singe a été décrit et l'infection est habituellement auto-limitation, avec une période d'incubation de 6 à 16 jours. Au Nigeria, le dernier cas d'infection au virus de la variole du singe a été enregistré plus de 46 ans. L'apparition récente dans le Nigeria s'est produite dans l'année 2017 et se serait étendue à 24 membres avec 228 individus présumés affectés. Le diagnostic de laboratoire, ainsi que la gestion et la prévention de l'infection au virus de la variole du singe au Nigeria, sont ambitieux que le Nigeria est un pays pauvre en ressources avec une infrastructure limitée, la compétence technique et de la formation qui est nécessaire pour faire un diagnostic. La capacité de la variole du singe Virus à évoluer, son potentiel potentiel bioterrorisme, ainsi que sa récente apparition au Nigeria est une raison supplémentaire de la nécessité d'améliorer la compréhension de la présentation et la prévention de l'infection au virus de la variole du singe.

Mots-clés : virus, virus émergents, le Nigeria, l'éruption, zoonose, orthopoxvirus

\section{ABSTRACT}

Monkeypox infection is a zoonotic infection transmitted by direct or indirect contact with blood, body fluids and lesions of an infected animal. Human to human spread of Monkeypox has been described and infection is usually self-limiting, with an incubation period of 6-16 days. In Nigeria, the last case of monkeypox infection was recorded over 46 years ago. The recent emergence in Nigeria occurred in the year 2017 and was reported to have spread to 24 states with 228 suspected individuals affected. Laboratory diagnosis, as well as management and prevention of monkeypox infection in Nigeria, remain challenging as Nigeria is a resource-poor country with limited infrastructure, technical skill and training which is required in making a diagnosis. The ability of the monkeypox virus to evolve, its potential bioterrorism potential, as well as its recent emergence in Nigeria further justifies the need for improving the understanding of the presentation and prevention of monkeypox infection Keywords: monkeypox, emerging virus, Nigeria, rash, zoonosis, orthopox

Copyright (C2017 AJCE M. This work is licensed under the Creative Commons Attribution 4.0 International License CC-BY. 


\section{INTRODUCTION}

Monkeypox is a relatively rare viral zoonotic infection which occurs primarily in remote parts of Central and West Africa, especially in the rural rainforest regions of Congo Basin and Democratic Republic of Congo, where it is considered to be endemic. It was first identified in 1958 during an investigation of a pox-like disease among monkeys in the State Serum Institute in Copenhagen, Denmark hence its name (1). The first case of human monkeypox infection was first described in a 9-year-old boy from Equateur province of Zaire, Democratic Republic of Congo, Central Africa, who developed a smallpox-like illness which was later confirmed as human monkeypox by World Health Organization (2).

Monkeypox virus is an oval brick-shaped virus with double-stranded DNA, which belongs to the Poxviridaefamily,thesubfamily; Chordopoxvirinae and genus Orthopoxvirus. Other members of the family include Variola virus, Vaccinia virus (the virus used in smallpox vaccine), Cowpox virus, Camelpox virus and Ectromelia virus ${ }^{3}$. Since the eradication of smallpox, monkeypox is the foremost Orthopoxvirus affecting man (4). There are two distinct clades of Monkeypox virus namely the Congo Basin and the West African clades; The Congo Basin clade is more virulent and has a higher rate of human-to-human transmission ${ }^{4-6}$.

The recent outbreak of monkeypox in Nigeria which spanned October 2017 to February 2018 was a source of public health concern. There was an initial diagnostic dilemma as many individuals; physicians inclusive had no knowledge of the aetiology of the disease condition. The Centre for Disease Prevention and Control (CDC) played a vital role in diagnosis, as well as implementation of treatment plan, prevention and control.

Monkeypox virus is a potential agent of bioterrorism as it is second to Variola virus, the agent of smallpox in terms of orthopoxvirus virulence with a mortality rate of $10 \%(3,5)$. Hence, there is an urgent need to educate physicians and the general public on the diagnosis, management and control of this disease.

\section{Biology of Monkeypox virus}

Monkeypox virus is an enveloped zoonotic virus and a species of the genus Orthopoxvirus in the family Poxviridae. It has a similar morphology, size, and genome to Variola virus; however, it has a very broad host range, making it capable of infecting many species such as rodents, monkeys, and humans. This has allowed the virus to persist in wild host reservoir, causing sporadic human diseases thus avoiding global eradication by vaccination. The monkeypox virus measures about 200-250nm in size, it appears brick shaped, with characteristic surface tubules and a dumb bell shaped core. Its genome is approximately $199 \mathrm{~kb}$ of double stranded linear DNA and contains approximately 190 non-overlapping Open reading frame (ORFs) $>180 \mathrm{nt}$ long (1,7-9,26). Like all orthopoxviruses, the central coding region sequence (CRS) at monkeypox virus nucleotide positions $56000-120000$ is highly conserved and flanked by variable ends that contain inverted terminal repeats (ITRs). At present, there are virtually no studies of the biology of the monkeypox virus as there have been no animal models in which to study monkeypox.

\section{EPIDEMIOLOGY}

Monkeypox is indigenous to the rainforests of Central and West Africa. Between 1970 and 1986, 10 cases of human monkeypox infection were reported in Sierra Leone, Nigeria, Liberia and Côte d'Ivoire all (5). A total of 338 cases were reported from surveillance reports from 1981 to 1986 in the Democratic Republic of Congo. Another outbreak occurred from 1996-1997 in the Democratic Republic of Congo, with an attack rate of 22 cases per 1000 population, sporadic occurrences in neighbouring countries have also been reported (5). The first report of human monkeypox infection outside Africa occurred in 2003 in the United States following shipment of rodents imported from Ghana to Texas. Eighty-one $(41 \%$ laboratory confirmed) cases of monkeypox were reported in this outbreak (6). In 2003, 11 cases of monkeypox and 1 death were reported from the Democratic Republic of Congo while in 2005, 10 cases were reported in Sudan.

Between September and December 2017, Nigeria reported 89 confirmed and 228 suspected cases of monkeypox from 24 out of the 36 states in the country (10). Studies of monkeypox virus have identified at least 2 different genetic types (clades) of the virus, both of which segregate based upon geographic separation, with one type being found in West Africa and the other in Central Africa (11). The West African clade was implicated in this outbreak. Most of the confirmed cases were among adults whose ages ranged from 21- 40 years with male to female ratio of 2.5:1. Six deaths were reported among which four reportedly had background immunosuppressive illness $(10,12$.) Prior to this, only 3 cases had been previously reported in Nigeria. The first reported case occurred in 1971, in a 4-year-old boy from the South Eastern part of the country while the last reported case occurred in 1978 (13). Prior to the recent outbreak, there had been no reported case of monkeypox in Nigeria since 1978. Another West African country, Liberia has also reported 2 confirmed cases of monkeypox in November 2017 14).

In endemic countries, transmission occurs all through the year without peak month or season 
(15). Incidence of monkeypox infection is equal in males and females with no racial predilection. In epidemics seen in Africa, children less than 15 years of age were the most affected and this might be linked to the cessation of smallpox vaccination (16).

\section{Pathogenesis}

Transmission to humans occurs via handling of infected animals, direct contact with the blood, body fluids, inoculation from mucocutaneous lesions on an infected animal, especially when the skin barrier is lost due to scratches, bites or other trauma or ingestion of inadequately cooked meat of infected animals $(5,15)$. Transmission has been documented through handling of infected monkeys, Gambian giant squirrels, rats, rabbits, dormice, porcupines, gazelles and prairie dogs (5). Outbreaks typically occur among inhabitants of small villages involved in hunting and gathering with close physical contact being the most significant risk factor for monkeypox infection. No animal has been identified as its exact natural reservoir however, rodents are being considered. Person to person transmission can occur via large respiratory droplets during prolonged face-to-face contact. Congenital monkeypox infection can also occur via placental transmission (5) .

Monkeypox infection begins with infection of the dermis or respiratory epithelium following transmission from infected animals or person respectively. Dissemination of the virus occurs through the lymphatics to the blood with resultant primary viraemia and systemic infection. Secondary viraemia occurs and results in infection of the epithelium with resultant skin and mucosal lesions. Replication of the virus in mucosal surfaces can result in its transmission via oropharyngeal secretions to close contacts (15). In spite of the host immune response, the density of virus in oropharyngeal secretions, proximity and duration of contact and virus survival likely affects the risk of transmission of the virus as the monkeypox virus has evolved mechanisms of evading the host immune response (15).

\section{Clinical manifestations}

Monkeypox has an incubation period which ranges from 4 to 21 days with an average of 6 to 16 days. This is followed by a prodromal illness which lasts for 1 to 5 days. Typical characteristics of the prodromal illness include fever $\left(38.5-40.5^{\circ} \mathrm{C}\right)$, malaise, intense headache, lymph node enlargement, back pain, myalgia and intense asthenia $(4,16)$. The prodromal phase is also known as the pre-eruptive phase and an individual may be infectious during this phase. Rashes appear within 1 to 10 days of development of fever, this is known as the eruptive or exanthema phase. The rash usually begins on the face and then spread to other parts of the body, the palms and the soles may also be affected. Affectation of the face is seen in $95 \%$ of cases (17). The lesions seen in monkeypox are extremely similar to that of smallpox: they appear monomorphic, hard and pea-sized on an erythematous base; often described as "dew drops on a rose petal"18. However, unlike smallpox, the lesions may appear in crops and do not have a strong centrifugal spread (5).

The sequence of appearance of the rash is as follows; macules, papules, vesicles and pustules involving both covered and uncovered areas. The rash evolves synchronously over 14 to 28 days, usually between 1-10 days after the onset of fever $(3,5)$. Some of the lesions may have a central umbilication. The lesions of chickenpox occur in successive crops with various stages of development being visible at any time hence aiding its differentiation from that of monkeypox, as these are usually in one stage of development and slowly progress to the next stage with each stage lasting 12 days $(4,17)$. Lesions may be few to several thousands in number, with affectation of the oral mucosa, genitalia and conjunctiva in $70 \%, 30 \%$ and $20 \%$ of cases respectively. Corneal affectation has also been described. Pruritus may be seen as well as petechiae and ulceration, pain is unusual. Crusting of the rash is seen in about 10 days after its onset and complete disappearance observed within 2-4 weeks of onset (17). An individual is infectious from the onset of exanthem through to the crusting of the rash. Fever subsides within 1-3 days after the onset of the rash, however, a second febrile period has been described when the lesions become pustular and this has been linked with deterioration in the general condition of the patient (4.)

Other symptoms seen include chills and/or drenching sweats, severe headache, backache, pharyngitis, myalgia, cough and shortness of breath. Enlargement of the submental, submandibular and cervical or inguinal lymph nodes is a common feature. The lymph nodes appear firm and sometimes tender. The presence of lymph node enlargement is a key feature in differentiation of monkeypox from smallpox and chickenpox, as it is a rare feature in smallpox while it is seen in $90 \%$ of unvaccinated patients with monkeypox infection $4,5,17$. Pitted scars and/or areas of hypo/hyperpigmentation may persist after crusts have fallen $(12,19)$.

Typically, human monkeypox infection is a selflimiting illness which lasts for 14-21 days. Severe cases are seen in children and may require intensive care and are usually related to the extent of virus exposure, health status, vaccination status, comorbidities and severity of complications $(5,17)$. Individuals previously vaccinated against smallpox have a milder form of the disease as they have fewer and smaller lesions, less lymphadenopathy 
and generally less severe disease $(4,16)$. Conditions which suppress cell-mediated immunity such as HIV may alter the natural history of the disease and may result in more severe infections (15). The lesions seen in them are also noticed to have regional monomorphism and a centrifugal distribution. Lesions in children may appear as nonspecific 1-5 $\mathrm{mm}$ erythematous papules, resembling an arthropod bite reaction. Mortality rates range from 1 to $10 \%$ with most cases occurring in children and in unvaccinated persons (16). Indirect or low-level exposure may result in asymptomatic or subclinical infections especially in persons living in or beside forests. Complications of monkeypox infection include pitted scars, deforming scars, secondary bacterial infection, keratitis, corneal ulceration, blindness, bronchopneumonia, septicaemia and encephalitis $(3,5,17)$.

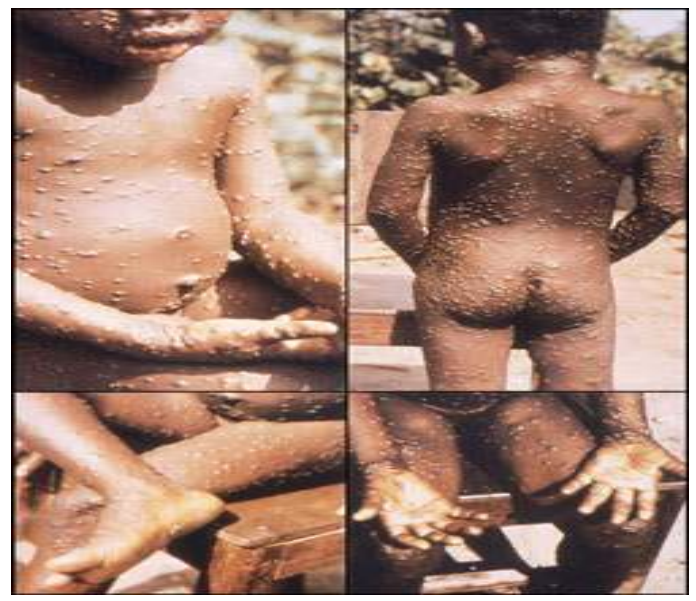

Figure 1: A child affected by monkeypox, showing generalized vesiculo-pustular rashes involving the trunk, limbs, face and limbs, extending to the palms and soles of the feet. Photo was reproduced from Centres for Disease Control and Prevention (https://www.cdc.gov/poxvirus/monkeypox/index.html)

\section{Laboratory diagnosis}

Diagnosis of monkeypox infection is made by cell culture, polymerase chain reaction (PCR), enzymelinked immunosorbent assay (ELISA) or Western blotting with PCR being used for definitive diagnosis ${ }^{3-5,18}$. Standard, contact and droplet precautions must be applied during specimen collection and all samples potentially infected with monkeypox virus should be handled in Biosafety level 2 facilities. Specimen that may be obtained to aid diagnosis include tonsillar tissue, oropharyngeal tissue or nasopharyngeal tissue swab, lesion biopsy using the punch biopsy kit, lesion fluid, lesion roof, scab/crust, whole blood, acute and convalescent phase sera $(4-6,16)$. Oropharyngeal or nasopharyngeal swab can be obtained for viral culture using MK2 cells, LLC-
MK2 cells and Vero E6 cells. Growth is detected by the presence of cytopathic effects which is seen as multinucleated syncytial keratinocytes. However, this cytopathic effect is also seen with Vaccinia and Cowpox viruses thereby causing a difficulty in differentiating these viruses via cell culture. The virus can be identified from the culture by DNA restriction analysis (5).

Exudates of the lesion on a swab, scabs of the lesion, skin biopsy of the vesiculopustular rash or a sample of the roof of an intact vesiculopustule can also be used for diagnosis. These can be analysed using PCR for DNA sequence-specific for monkeypox virus such as the monkeypox extracellular-envelope virus protein gene and the conserved segment of the DNA polymerase gene (E9L) $(19,20)$. Maintenance of a cold chain upon specimen collection is preferable but if cold chain is unavailable, the viral DNA in the specimen is stable for a long time if kept in a cool, dark environment. Detection of antibodies; immunoglobin M (IgM) or immunoglobin $\mathrm{G}$ (IgG) in serum or plasma using ELISA is an effective means of diagnosis of monkeypox infection (21). IgM is detected in serum about 5 days after the onset of rash while IgG is detected after more than 8 days of the onset of rash. Paired sera for IgG and IgM titers may be analyzed for increasing titre values to detect seroconversion which is used as an indicator of recent monkeypox infection (21). Tzanck smear can be used to differentiate monkeypox infection from other nonviral disorders but it does not differentiate monkeypox infection from smallpox or herpetic infections. Antibody Immuno Column for Analytical Processes (ABICAP), an immunefiltration technique which works based on gravitydriven flow-through antigen capture ELISA has been developed. It is an on-site laboratory diagnostic test which can be used in both humans and animals (22).

Biopsy specimen from lymph nodes or scab material; vesicular fluid, blood specimen or viral culture can be examined using electron microscopy with negative staining revealing a large brickshaped particle with rounded corners which is characteristic of a poxvirus (18). Round-to-oval intracytoplasmic inclusions with sausage-shaped structures centrally measuring about 200-300 $\mu \mathrm{m}$ may also be seen on electron microscopic examination. These inclusions are commonly found with Orthopox viruses thereby aiding differentiation from Herpes and Parapox viruses (5).

Histological examination of papules may reveal acanthosis, individual keratinocyte necrosis and basal vacuolation along with a superficial and deep perivascular, lymphohistiocytic infiltrate in the dermis $(5,20)$. Histology of the vesicles may reveal spongiosis with ballooning and reticular 
degeneration. Pustules may reveal necrosis of the epidermis with numerous eosinophils and neutrophils, with many displaying karyorrhexis. The necrosis may extend through the entire thickness of the epidermis with a sharp lateral demarcation from adjacent intact epidermis. An associated perivascular infiltrate consisting of eosinophils and neutrophils in addition to lymphocytes and histiocytes may indicate secondary vasculitis. Presence of viral inclusions may be suggested by the presence of amphophilic intranuclear structures within the keratinocytes ${ }^{5}$. Eosinophilic Guarnieri-type intracytoplasmic inclusions are also seen within the keratinocytes (20). Orthopox viral antigens can be assessed using immunohistochemistry staining but its availability is limited to select reference laboratories.

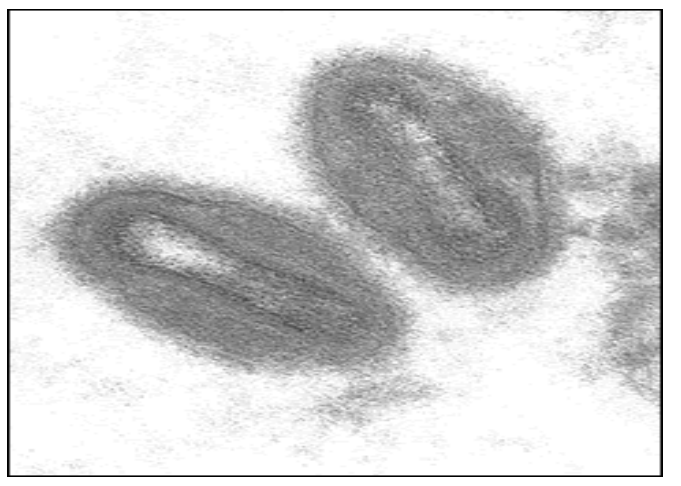

Figure 2: Electron micrograph of monkeypox virus. Reproduced from:

http://www.uwlax.edu/clinmicro/clinicalmicro.htm University of Wisconsin-La Crosse, Microbiology program (No Author Specified).

\section{Case Management}

Monkeypox is self-limiting hence treatment is mainly by bed rest and supportive care. However, hospitalization and possible intensive care may be necessary in severe cases. Preferably, nursing should be done in a negative pressure room while applying airborne and contact precautions to limits its spread. Isolation of infected individuals is also a necessary precaution in preventing the spread of the infection and this must be continued until the last crust is shed as direct contact with skin lesions and fomites are considered infectious (6).

There is no proven treatment for human infection at the moment but cidofovir and brincidofovir (CMX001) have been shown to have anti-monkeypox viral activity in vitro and in animal studies $(3,6)$. A reduction in mortality was observed with the use of cidofovir than therapeutic use of smallpox vaccine following intratracheal infection of cynomolgus monkeys (23). However, CDC recommends consideration of cidofovir in individuals with severe monkeypox infection; brincidofovir has an improved safety profile over cidofovir as it has less renal toxicity during its use in the treatment of cytomegalovirus infection. Tecovirimat (previously known as ST-246) is an oral antiviral agent with activity against orthopoxviruses including monkeypox in vitro and in animal studies but its effectiveness in humans is unknown (24).

Vaccinia Immune Globulin (VIG) is a blood product which is rich in antibodies against Vaccinia virus; it is obtained from pooled blood of individuals who have been inoculated with the smallpox vaccine. There is no available data on the effectiveness of VIG on the prevention and treatment of complications from monkeypox infection, however its use may be considered in patients with severe infection ${ }^{15}$. CDC recommends the prophylactic use of VIG in persons who have been exposed to the virus but have severe cellular immunodeficiency with contraindication to smallpox vaccination (6).

\section{Prevention and Control}

Restriction of movement of monkeys and small African mammals may limit the spread of monkeypox virus outside Africa ${ }^{6,25}$. Animals with suspected monkeypox infection which may be demonstrated as rhinorrhea, respiratory distress, mucocutaneous lesions, ocular discharge and/or lymphadenopathy should be quarantined while avoiding contact especially bites and scratches and exposure to body fluids and secretions. Animals that might have come in contact with an infected animal must be quarantined and observed for symptoms of monkeypox for 30 days. Use of gloves and wearing of protective clothing while handling sick animals or during slaughtering procedures, thorough cooking of all animal products before eating also limits the risk of infection ${ }^{6,25}$. There is need to avoid contact with any material that has been in contact with infected animal, practicing good hand hygiene after contact with infected animals or persons also limits the risk of infection and its spread (5).

There is currently no commercially available vaccine specific for monkeypox. Immunization of healthcare workers and those exposed to their samples with the smallpox vaccine such as ACAM2000, which contains live Vaccinia virus confers $85 \%$ protection from monkeypox infection ${ }^{4}$. Subsequent monkeypox infection may be milder even several years post vaccination with a reduction in the incidence of complications (16). The investigational vaccines Aventis Pasteur Smallpox Vaccine (APSV) which contains a replication-competent Vaccinia virus and Imvamune (MVA-BN): a replication-deficient smallpox vaccine which contains an attenuated live virus are also available but have restricted use ${ }^{6}$. Imvamune may be used in individuals with certain immune deficiencies. The vaccine, LC16m8 which also contains attenuated vaccinia virus and has less adverse effects than ACAM2000 has been licensed 
for used in Japan (4.) Prior vaccination with vaccinia virus is known to provide $85 \%$ protection and reduce the severity of the infection but caution is needed in population with high HIV prevalence ${ }^{3,4}$. The discontinuation of the general smallpox vaccination and the ability of the virus to evolve may have contributed to increased susceptibility of humans to monkeypox infection $(4,15)$.

In the case of significant unprotected exposure to an infected animal or person, CDC recommends preexposure smallpox vaccination to field investigators, laboratory personnel, veterinarians, and healthcare workers investigating or caring for patients with suspected monkeypox. Additionally, post-exposure smallpox vaccination within two weeks of exposure preferably within 4 days of exposure is preferable (6). Due to the relative rarity of monkeypox infection, vaccination of an entire population is uncommon and caution must be applied when considering vaccination in populations with high prevalence of HIV infection due to the risk of complications (5).

Challenges with Diagnosis, Management and Prevention of Monkeypox in Nigeria

It is over 46 years since the first case report of monkeypox in Nigeria, however there is still limited public awareness of the disease. Laboratory diagnosis, as well as management and prevention of monkeypox infection in Nigeria remain challenging. Nigeria is a resource-poor country with limited infrastructure, technical skill and

\section{REFERENCES}

1. Von Magnus P, Andersen E, Petersen K, BirchAndersen A. A pox-like disease in cynomolgus monkeys. Acta Pathol Microbiol Immunol Scand 1959; 46: 156-176

2. Landyi I, Ziegler P, Kima A. A human infection caused by monkeypox virus in Basankusu Territory, Democratic Republic of Congo (DRC). Bull World Health Organ 1972; 46: 593-597.

3. Weinstein R, Nalca A, Rimoln A, Bavari S, Whitehouse C. Reemergence of Monkeypox: Prevalence, Diagnostics, and Countermeasures. Clin Infect Dis 2005; 41: 1765-1771.

4. McCollum A, Damon I. Human Monkeypox. Clin Infect Dis 2014; 58: 260-267.

5. Pal M, Mengstie F, Kandi V. Epidemiology, Diagnosis and Control of Monkeypox Disease: training which is required in making a diagnosis. This might be attributed to the relative rarity of the disease as well as poor political willpower in health-related matters. There is a need to increase public awareness among the populace, physicians inclusive by dissemination of accurate information through authentic platforms on the risk factors, symptoms, signs, prevention and control of the disease. Emphasis should be made on contact precautions while handling animals and thorough cooking of animals before ingestion. There is also a need to clarify myths about monkeypox disease in the various communities and states. Furthermore, access to basic health care should be provided for all through health insurance schemes in order to aid early diagnosis, treatment and control of the infection. Improvement of political willpower in health-related matters via policy making, provision of funds for infrastructure, personnel training and research is also necessary. Development of a fielddeployable point-of-care test further aiding the diagnosis is important.

\section{CONCLUSION}

In conclusion, monkeypox virus infection is relatively rare and usually self-limiting. In the era of globalization, with consequent increased mobility of man and cross-border transport of animals, there is a potential for the spread of monkeypox virus to several parts of the world. The ability of the virus to evolve, the potential threat of its use in bioterrorism, as well as its re-emergence in Nigeria further justify the need for better understanding of monkeypox infection.

A comprehensive Review. Am J Infect Dis Microbiol 2017; 5: 94-99.

6. Monkeypox. Cent Dis Control Prev 2018.https://www.cdc.gov/poxvirus/monkey pox/.

7. Moss B. Poxviridae: The viruses and their replication. In: Knipe DM, Howley PM. Field's Virology, 4th ed. Philadelphia: LippincottRaven 2001. pp. 2849-83.

8. Hendrickson RC, Wang C, Hatcher EL, Lefkowitz EJ. Orthopoxvirus genome evolution: the role of gene loss. Viruses. 2010; 2:1933-67.

9.

Shchelkunov SN, Totmenin AV, Babkin IV, Safrono v PF, Ryazankina OI, Petrov NA, Human monkeypox and smallpox viruses: genomic comparison. FEBS Lett. 2001;509:66-70. D

10. Monkeypox outbreak in Nigeria. Niger Cent Dis Control

2018.http://www.ncdc.gov.ng/diseases/sitre 
ps $/$ cat $=8 \&$ name $=$ An $\% 20$ Update $\% 20$ of $\% 20 \mathrm{Mo}$ nkeypox\%20Outbreak\%20in\%20Nigeria.

11. Chen N, Li G, Liszewski M, Atkinson J, Jahrling P, Feng $\mathrm{Z}$ et al. Virulence differences between monkeypox virus isolates from West Africa and the Congo basin. Virology 2005; 340: 46-63.

12. Yinka-Ogunleye A, Aruna O, Ogoina D, Aworabhi N, Eteng W, Badaru S et al. Reemergence of human monkeypox in Nigeria, 2017. Emerg Infect Dis 2017; 24.

13. Eke R. Monkeypox in a four year old girl: case report. West Afr Med J Niger Pract 2017; 1: 2122.

14. ProMED-mail. https://www.promedmail.org/ (accessed 7 May2018).

15. Sutcliffe C, Rimoin A, Moss WJ. Viral Infections with Cutaneous lesions: Poxviruses. In: Hunter's Tropical Medicine and Emerging Infectious Disease. Elsevier, 2012, pp 256-262.

16. Jezek Z, Fenner F. Human Monkeypox. In: Monographs in Virology. Karger: Basel, Switzerland, 1988.

17. Huhn G, Bauer A, Yorita K, Graham M, Sevar J, Likos A et al. Clinical characteristics of human monkeypox and risk factors for severe disease. Clin Infect Dis; 41: 1742-1751.

18. Mullendore NF, Lawner BJ, Malone JD. Monkeypox Attack. In: Ciottone's Disaster Medicine. Elsevier, 2016, pp 774-776.
19. Breman J., Kalisa-Ruti, Steniowski M., Zanotto E, Gromyko A I, Arita I. Human monkeypox, 1970-1979. Bull World Health Organ 980; 58: 165-182.

20. Bayer-Garner IB. Monkeypox virus: histologic, immunohistochemical and electronmicroscopic findings. J Cutan Pathol 2005; 32: 28-34.

21. Karem K, Reynolds M, Branden Z, Lou G, Bernard N, Patton J. Characterization of acutephase humoral immunity to monkeypox: use of immunoglobulin $\mathrm{M}$ enzyme-linked immunosorbent assay for detection of monkeypox infection during 2003 North American outbreak. Clin Diagn Lab Immunol 2005; 12: 867-872.

22. Stern D, Pauly D, Zydek M, Miller L, Piesker J, Lisdat $\mathrm{F}$ et al. Development of a genus-specific antigen capture ELISA for Orthopoxviruses Target Selection and Optimized Screening. PLOS ONE; 11: e0150110.

23. Stittelaar K, Neyts J, Naesens L, van Lavieren R, Holý A, De Clercq E et al. Antiviral treatment is more effective than smallpox vaccination upon lethal monkeypox virus injection. Nature 2006; 439: 745-748.

24. Jabeen C, Umbreen G. Monkeypox transmission, need and treatment of humans with an antiviral drug. Int J Soc Sci Manag 2017; 4: 77-79.

25. Monkeypox. World Health Organ. http://www.who.int/news-room/factsheets/detail/monkeypox (accessed 8 May2018). 